\title{
QUANTIFICAÇÃO DA FALHA NA MADEIRA EM JUNTAS COLADAS UTILIZANDO TÉCNICAS DE VISÃO ARTIFICIAL ${ }^{1}$
}

\author{
Christovão Pereira Abrahão ${ }^{2}$, Carlos Alberto Alves Varella ${ }^{3}$, Francisco de Assis de Carvalho Pinto ${ }^{4}$ e \\ Joseph Kalil Khoury Junior ${ }^{5}$
}

\begin{abstract}
RESUMO - Com o emprego de adesivos pode-se obter um grande número de produtos derivados da madeira. Para confecção industrial de produtos de madeira colada, normas reconhecidas internacionalmente exigem que a adesão da madeira seja testada segundo procedimentos padronizados e que nos resultados destes testes seja reportado, além da resistência das juntas, o porcentual de falha na madeira. Para avaliação da falha a norma ASTM D526699 recomenda o emprego de uma rede de quadrículas traçada sobre um material transparente. Contudo, esta avaliação, além de demandar muito tempo, ainda é realizada com muita subjetividade. A hipótese do presente trabalho é que se pode quantificar a falha na madeira com um sistema de visão artificial, tornando o procedimento mais rápido e menos sujeito à subjetividade. Foram testados dois tipos de algoritmos de limiarização automática em imagens adquiridas com digitalizadores de mesa. Concluiu-se que a falha na madeira pode ser quantificada por limiarização automática em substituição ao método convencional das quadrículas. Os algoritmos testados apresentaram erro médio absoluto menor que 3\% em relação ao sistema convencional da rede quadriculada.
\end{abstract}

Palavras-chave: Processamento de imagens, madeira, adesão e automação.

\section{MEASURING WOOD FAILURE PERCENTAGE USING A MACHINE VISION SYSTEM}

\begin{abstract}
It is possible to obtain several products by glueing wood. Internationally approved standards require wood adhesion to be tested according to standardized procedures, including in the results, shear stress and wood failure percentages. In order to estimate wood failure percentage, the ASTM D5266-99 standard suggests the use of a grid template printed on a transparent sheet. However, this evaluation is not only time-consuming but also subjective. This work developed and tested an algorithm to quantify the flawed wood areas by using a machine vision system, a faster and less subjective procedure. Two types of automatic threshold algorithms were tested. The glued wood samples were scanned after the shear tests under compression. It was concluded that automatic measurement can replace the manual grid method. The proposed algorithms presented an average absolute error of $3 \%$, as compared to the manual grid method.
\end{abstract}

Key words: Image processing, wood, adhesion, and automation.

\section{INTRODUÇÃO}

A capacidade de adesão da madeira constitui uma das mais importantes propriedades tecnológicas deste material. Com o auxílio de adesivos pode-se obter um grande número de produtos derivados da madeira. Por exemplo, peças de dimensões limitadas podem ser utilizadas na confecção de gigantescos elementos estruturais na forma de madeira laminada colada. As normas norteamericanas D905-98 - Standard test method for strength

1 Recebido para publicação em 4.4.2002.

Aceito para publicação em 19.2.2003.

Pesquisa desenvolvida com apoio financeiro da FAPEMIG.

2 Doutorando em Ciência Florestal, Dep. de Engenharia Florestal da Universidade Federal de Viçosa - UFV <cabrahao@vicosa.ufv.br>; ${ }^{3}$ Professor Assistente UFRRJ, Doutorando em Engenharia Agrícola, Dep. de Engenharia Agrícola da UFV, <varella@vicosa.ufv.br>; ${ }^{4}$ Prof. Adjunto, Dep. de Engenharia Agrícola da UFV, <facpinto@ufv.br>; ${ }^{5}$ Doutorando Engenharia Agrícola, Dep. de Engenharia Agrícola da UFV, <khouryjr@vicosa.ufv.br>. 
properties of adhesive bonds in shear by compression loading e D2339 - 98 - Standard test method for strength properties of adhesives in two-ply wood construction in shear by tension loading versam sobre os procedimentos para determinação da capacidade de adesão da madeira e ambas exigem que entre os resultados dos testes sejam reportados a resistência das juntas coladas e o porcentual de falha na madeira, bem como a variabilidade destes resultados (ASTM, 2000). Ainda segundo as normas ASTM D2559-99 - Standard specification for adhesives for structural laminated wood products for use under exterior (wet use) exposure conditions e D5771-99 Standard specification for adhesives used for laminated joints in nonstructural lumber products - que fornecem, respectivamente, as especificações exigidas para os usos estrutural e não-estrutural de componentes de madeira colada, as amostras testadas devem apresentar pelo menos $75 \%$ de falha na madeira no primeiro caso e $60 \%$ no segundo. Valores altos para o porcentual de falha na madeira são indicativos de boa qualidade na adesão, pois demonstram que a coesão do adesivo e a resistência da interface adesivo-madeira são mais altas que a resistência da própria madeira (ASTM, 2000). Assim, o dimensionamento de produtos em que sejam empregadas colagens pode utilizar com segurança as propriedades da madeira como base para cálculos. Por outro lado, falhas baixas indicam que a utilização da madeira não está sendo otimizada com a aplicação do adesivo em teste, já que a ruptura ocorre com tensões menores que a resistência da madeira. Em última instância, a reprodutibilidade dos resultados de resistência das juntas e do porcentual de falha na madeira é que indicará a adequação do uso de um determinado adesivo para a colagem de uma determinada espécie de madeira. Investigações acerca das condições de colagem como temperatura, pressão, tratamento das superfícies, entre outros, podem melhorar o desempenho da adesão (Della Lucia \& Vital, 1981).

A norma D5266-99 - Standard practice for estimating the percentage of wood failure in adhesive bonded joints (ASTM, 2000) descreve a técnica de medição das áreas de falha na madeira. Nessa técnica empregase uma rede de quadrículas traçada sobre um material transparente. Contudo, esta avaliação, além de demandar muito tempo, ainda é realizada com muita subjetividade.

Em diversas áreas do conhecimento têm-se empregado técnicas de processamento e análise de imagens, visando a automatização de rotinas laboratoriais, o controle de qualidade na indústria etc. Esses sistemas de

R. Árvore, Viçosa-MG, v.27, n.1, p.71-78, 2003 visão artificial, na maioria dos casos, utilizam a segmentação do objeto de interesse na imagem original como uma das primeiras etapas no processamento da imagem. Diversas são as técnicas de segmentação utilizadas com base no valor numérico de cada pixel, na análise de textura e no histograma da imagem (Gonzalez \& Woods, 2000). A limiarização, que tem sido a mais utilizada, visa a obtenção de uma imagem binária, segmentando a cena original em objetos de interesse e fundo com base em um determinado valor da escala de cinza, o limiar. A definição desse limiar é dependente das características da cena. Diferentes algoritmos para determinação automática desse limiar têm sido propostos e testados.

Yang et al. (2001) testaram o desempenho de quatro algoritmos para estimar a distribuição da biomassa em membranas biológicas heterogêneas. Han et al. (1990) desenvolveram um procedimento para análise de imagem com objetivo de determinar a cobertura do solo. Lira et al. (2000) usaram imagens digitalizadas coloridas de 24-bit com resolução de 100 dpi para avaliar o número de nódulos em leguminosas. Haney et al. (1994) construíram um protótipo de uma máquina equipada com visão artificial para classificar tábuas. Quin et al. (1998) utilizaram uma câmera com sensibilidade para o infravermelho com o objetivo de identificar nós em tábuas de pinhoamarelo.

A hipótese testada no presente trabalho é de que é possível quantificar a falha na madeira em um ensaio da resistência de juntas coladas com um sistema de visão artificial, obtendo-se com esta técnica resultados equivalentes àqueles obtidos pelo método convencional da rede de quadrículas. A quantificação da falha na madeira com base em imagens digitais pode ser um procedimento mais rápido e menos sujeito à subjetividade do que o método convencional das quadrículas. Acredita-se que para esse tipo de classificação as imagens adquiridas com digitalizadores de mesa (scanner) apresentam algumas vantagens em relação às fotográficas, visto que estes equipamentos possuem iluminação própria e padronizada, são de fácil operação, estão menos sujeitos a distorções geométricas e são de menor custo. Porém, os digitalizadores de mesa apresentam diferentes especificações e possibilidades de ajustes, dependendo do modelo e da marca, que podem influenciar o desempenho dos algoritmos de processamento e a análise das imagens (Vieira-Junior et al., 1999).

O desenvolvimento de uma técnica que venha a conferir maior rapidez e exatidão à avaliação da falha na 
madeira pode trazer benefícios para as pesquisas relacionadas à adesão da madeira e para as indústrias de derivados da madeira que empregam adesivos em seus produtos, como na fabricação de compensados, painéis sarrafeados e madeira laminada colada. Tais indústrias necessitam realizar este tipo de teste no controle de qualidade de seus produtos, e a disponibilidade de equipamentos e programas de computador especialmente projetados para determinação da falha na madeira pode ser de grande auxílio.

Portanto, os objetivos gerais do presente trabalho foram desenvolver e testar um método para quantificação da área de falha na madeira, utilizando técnicas de processamento e análise de imagens digitais, tendo como padrão comparativo o método convencional das quadrículas. Os objetivos específicos foram testar dois tipos de algoritmos de limiarização automática e três tipos de imagens (resolução e equipamento).

\section{MATERIAL E MÉTODOS}

\subsection{A Madeira e o Adesivo}

Nos ensaios de adesão utilizou-se a madeira de Pinus elliottii Engelm. As peças usadas eram totalmente isentas de defeitos, tais como nós, bolsas de resina, rachaduras e outros. Foi empregado o adesivo à base de resorcinolformaldeído sob o nome comercial Cascophem 200, da Alba Química S.A., aplicado na razão de $300 \mathrm{~g}$ da mistura adesiva por metro quadrado de superfície de madeira, conforme recomendação do fabricante. A pressão de colagem aplicada foi de $10 \mathrm{kgf} / \mathrm{cm}^{2}$, por 8 horas. Após a completa cura do adesivo (uma semana depois da retirada da prensa), as juntas foram recortadas para obtenção dos corpos-de-prova. Obtiveram-se cinco corpos-de-prova por cada junta, os quais foram testados no cisalhamento por compressão segundo a norma D905-98 (ASTM, 2000). Em seguida, 50 corpos-de-prova falhados foram recortados, as dimensões de suas faces foram padronizadas em 4,5 x 4,5 cm e, então, obtidas as imagens.

\subsection{Aquisição das Imagens}

Foram adquiridas imagens coloridas e em tons de cinza dos 50 corpos-de-prova preparados com dois modelos de digitalizadores da marca Genius. Com o modelo Color Page-I foram adquiridas 50 imagens em 256 tons de cinza ( 8 bits), com resolução de 100 dpi. Com o modelo Color Page Vivid III foram obtidas
50 imagens coloridas e com resolução de 150 dpi e outras 50 imagens em 256 tons de cinza ( 8 bits) com resolução de 100 dpi.

Para obtenção das imagens, as mesas dos digitalizadores foram cobertas com uma máscara de papel cartão branco, contendo uma janela para posicionamento das amostras e com as mesmas dimensões destas $(4,5 \times 4,5 \mathrm{~cm})$. O uso da máscara teve como objetivos evitar a entrada da luz ambiente no interior do digitalizador e fixar a posição das amostras na mesa de vidro, facilitando o trabalho de digitalização.

\subsection{Avaliação das Áreas Falhadas pelo Método Convencional}

O método convencional teve como base a norma D5266-99 (ASTM, 2000). Essa norma não define qual deve ser o formato das redes de quadrículas, porém diz que deve ser empregada uma régua com divisões de 2,5 $\mathrm{mm}$ ou, ainda, para auxiliar o trabalho, pode-se utilizar uma rede com subdivisões que representem porcentuais conhecidos da área total colada do corpode-prova. Neste trabalho utilizou-se uma rede de quadrículas que combinou as duas recomendações da norma, ou seja, divisões de $2,5 \mathrm{~mm}$ e agrupamentos de nove quadrículas evidenciados por linhas mais espessas. A rede de quadrículas foi fixada a um gabarito, para facilidade de posicionamento das amostras (Figura 1). Nesse método as áreas foram quantificadas por dois avaliadores treinados.

\subsection{Avaliação das Áreas Falhadas por Limiarização Automática}

Para automatização da leitura do ensaio, foram implementados e testados dois algoritmos de segmentação, preparados para detecção automática dos limiares. Um deles, construído pelos autores, baseou-se na estatística bayeseana, e será denominado neste trabalho como "bayeseano", o outro foi extraído do trabalho de Ridler \& Calvard (1978), citados por Yang et al. (2001), e será denominado de "iterativo".

A determinação automática do limiar pelo método bayeseano procura segmentar a imagem em duas classes de valores de brilho dos pixels com base em seu histograma. O histograma é considerado como a soma de duas funções de distribuições normais univariadas. A definição do limiar é realizada de tal maneira que se minimize a

R. Árvore, Viçosa-MG, v.27, n.1, p.71-78, 2003 
probabilidade do erro de classificação, que é equivalente à solução, para a variável $\mathrm{T}$ da equação a seguir (Reid et al., 1985; Gonzalez \& Woods, 2000):

$$
\left(\sigma_{1}^{2}-\sigma_{2}^{2}\right) T^{2}+2\left(\mu_{1} \sigma_{2}^{2}-\mu_{2} \sigma_{1}^{2}\right) T+2 \sigma_{1}^{2} \sigma_{2}^{2} \ln \left(\frac{P_{1} \sigma_{2}}{P_{2} \sigma_{1}}\right)+\mu_{2}^{2} \sigma_{1}^{2}-\mu_{1}^{2} \sigma_{2}^{2}=0
$$

em que $\mathrm{T}=$ valor do limiar ótimo; $\mu_{1}$ e $\mu_{2}=$ valores das médias dos níveis de cinza de cada classe; $\sigma_{1}^{2}$ e $\sigma_{2}^{2}=$ valores das variâncias dos níveis de cinza de cada classe; e $\mathrm{P}_{1}$ e $\mathrm{P}_{2}=$ são as probabilidades a priori da ocorrência de cada classe.

Dessa maneira, para determinação do limiar ótimo (T) deve-se ter conhecimento dos parâmetros da distribuição (média e variância) e da probabilidade de ocorrência de cada classe. Esses parâmetros foram determinados por método numérico iterativo de minimização do erro entre o histograma atual e a distribuição normal bimodal, método de Cauchy ou máximo declive (Mateus \& Luna, 1986). A Figura 2 apresenta a imagem de uma amostra com seu respectivo histograma bimodal, onde os picos caracterizam as populações de pixels pertencentes às regiões de falha na madeira (valores mais altos) e falha na cola (valores mais baixos). O limiar ótimo encontra-se em um ponto situado no vale entre os picos.

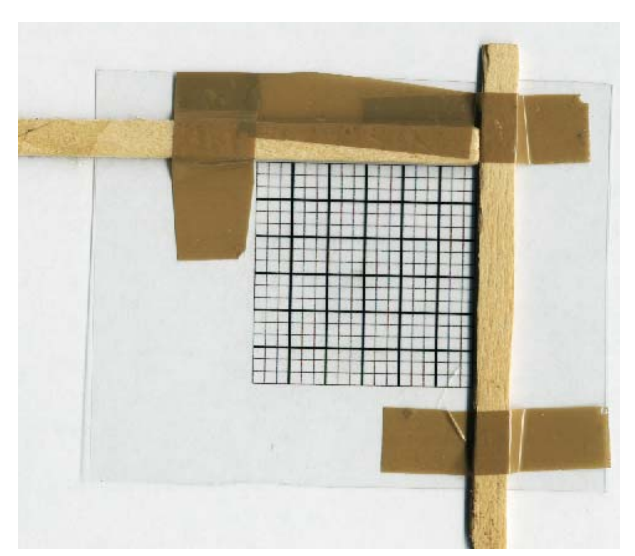

Figura 1 - Gabarito contendo a rede de quadrículas utilizada para avaliação da falha na madeira pelo método convencional.

Figure 1-Template with the grid used to evaluate the wood failure by the conventional method.

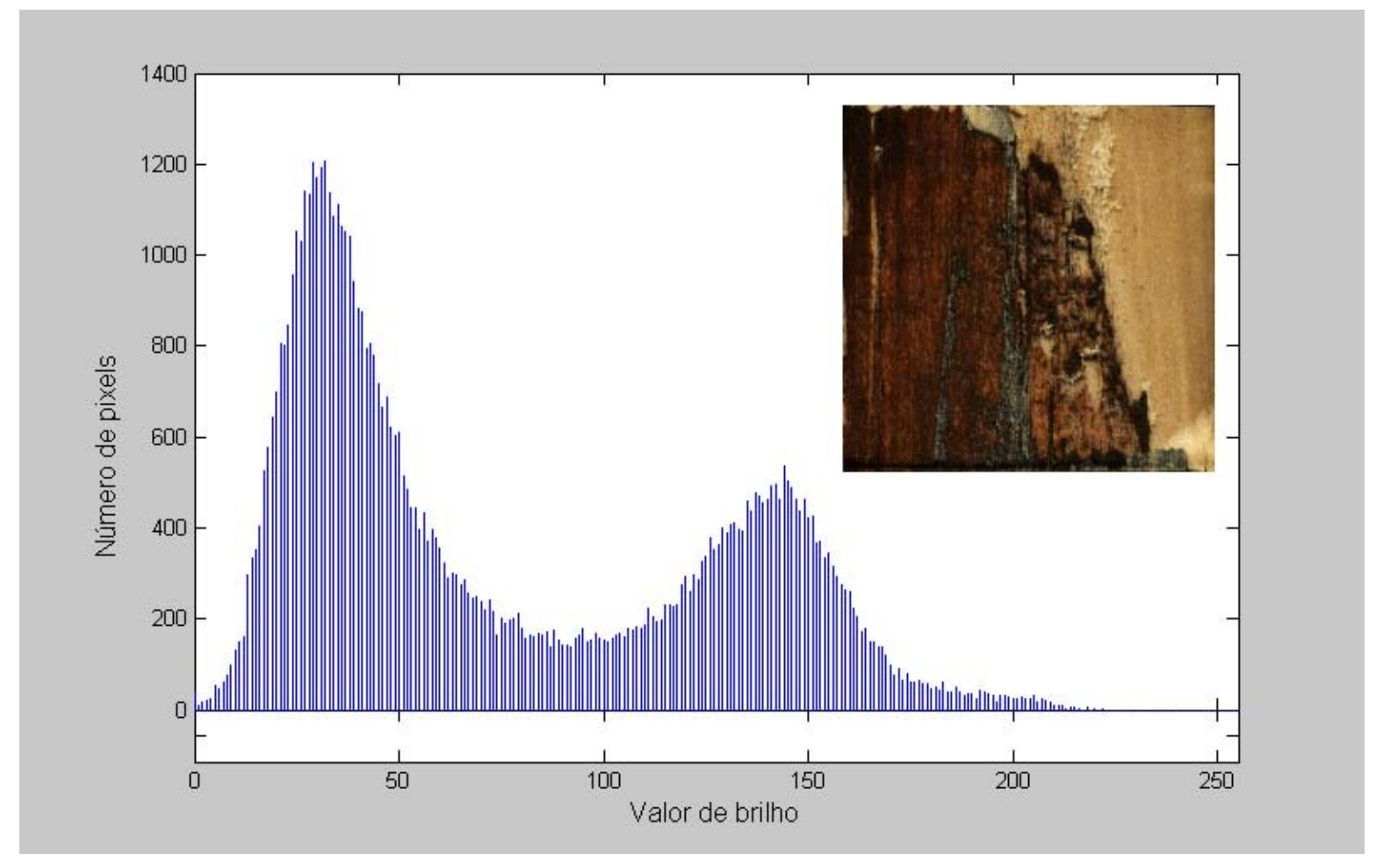

Figura 2 - Imagem de uma amostra com o seu respectivo histograma bimodal. Figure 2 - Image of a sample with its respective bimodal histogram. 
O método iterativo (Yang et al., 2001) também assume a existência de duas classes e que o limiar ótimo é igual à média das médias dos níveis de cinzas dessas classes. Portanto, o método realiza uma procura exaustiva desse limiar a partir do valor zero até o nível de cinza que divide o histograma em duas classes e atende ao critério preestabelecido. Este método tem a vantagem de apresentar o tempo de processamento bem menor que o do bayeseano, porém não é ótimo, pois não minimiza o erro de classificação.

\subsection{Avaliação dos Algoritmos}

A média aritmética dos resultados obtidos por dois avaliadores humanos, no método convencional, foi considerada como padrão na comparação com os resultados obtidos pelos algoritmos bayeseano e iterativo.

A comparação entre os métodos convencional e proposto foi realizada por meio de análises de regressão e do erro absoluto médio. Utilizou-se a regressão linear simples entre os resultados obtidos pelo método convencional e os resultados obtidos pelos algoritmos. Foi adotado o modelo de regressão sem intercepto, e considerou-se como o melhor resultado aquele que apresentou valor do estimador do coeficiente angular mais próximo da unidade, maior coeficiente de determinação $\left(r^{2}\right)$ e menor erro absoluto médio.

O erro médio absoluto (EMA) foi calculado por meio da expressão:

$$
\mathrm{EMA}=\frac{1}{\mathrm{n}} \sum_{\mathrm{i}=1}^{\mathrm{n}}\left\|\mathrm{x}_{\mathrm{i}}-\mathrm{y}_{\mathrm{i}}\right\|
$$

em que EMA = erro médio absoluto $(\%) ; x_{i}=$ valores de porcentagem de falha na madeira obtidos por meio da avaliação convencional $(\%) ; y_{i}=$ valores de porcentagem de falha na madeira obtidos pelo algoritmo testado (\%); e $\mathrm{n}=$ número de observações.

A nomenclatura utilizada para cada tratamento (combinação do tipo de imagem, equipamento e algoritmo) é apresentada no Quadro 1.

\section{RESULTADOS E DISCUSSÃO}

A Figura 3 ilustra o aspecto das superfícies de dois corpos-de-prova após o teste de cisalhamento em juntas coladas, evidenciando dois casos extremos de alta e baixa falhas na madeira ( $a$ e b, respectivamente). Neste ponto é importante ressaltar que a madeira empregada apresenta coloração clara e que a resina, após a cura, assume cor escura, situação esta bastante favorável aos propósitos deste trabalho. O emprego de corantes que não interfiram nas propriedades dos adesivos pode ser a solução para o pequeno contraste encontrado em algumas combinações adesivo-madeira.

Os algoritmos propostos segmentaram os pixels da imagem original em duas classes. Os pixels com valores de brilho menores do que o limiar ótimo foram considerados pertencentes ao adesivo e receberam o valor "zero" - áreas escuras, já os de valores de brilho maiores foram atribuídos à falha na madeira e receberam o valor " 1 " áreas claras. A Figura 3 apresenta duas imagens originais ( $a$ e b) e binárias (c e d) de corpos-de-prova com alto e baixo porcentuais de falha na madeira. A porcentagem de falha madeira foi obtida pela relação entre o número de pixels brancos e pixels totais na imagem binarizada.

No Quadro 2 estão os valores do estimador do coeficiente angular $(\hat{\beta})$, da diferença absoluta entre este estimador e a unidade (DA), do coeficiente de determinação $\left(\mathrm{r}^{2}\right)$ e do erro médio absoluto (EMA) obtidos nos testes dos algoritmos. Verifica-se que o algoritmo bayeseano apresentou o melhor resultado nas imagens coloridas adquiridas com o digitalizador de mesa Color Page Vivid III, isto é, valor de DA igual a $0,0117, \mathrm{r}^{2}$ de 0,9924 e EMA de 3,94\%. O algoritmo iterativo apresentou menor DA $(0,0159)$ e menor $\operatorname{EMA}(3,19)$ nas imagens adquiridas em tons de cinza com o digitalizador Color Page Vivid III, e maior $\mathrm{r}^{2}$ nas imagens coloridas com esse mesmo digitalizador.

O resultado da análise de variância para os erros médios absolutos é apresentado no Quadro 3. Verificase que houve diferença significativa entre os tratamentos a $1 \%$ de probabilidade, pelo teste $\mathrm{F}$.

Quadro 1 - Nom enclatura utilizada para os tratam entos testados

Table 1 - T erm inology used for the tested treatm ents

\begin{tabular}{|c|l|l|l|}
\hline Tratamento & Algoritmo & \multicolumn{1}{|c|}{ Imagem } & \multicolumn{1}{|c|}{ Digitalizador } \\
\hline 1 & Bayeseano & 256 tons de cinza & Color Page I \\
2 & Bayeseano & 256 tons de cinza & Color Page Vivid III \\
3 & Bayeseano & Colorida & Color Page Vivid III \\
4 & Iterativo & 256 tons de cinza & Color Page I \\
5 & Iterativo & 256 tons de cinza & Color Page Vivid III \\
6 & Iterativo & Colorida & Color Page Vivid III \\
\hline
\end{tabular}

R. Árvore, Viçosa-MG, v.27, n.1, p.71-78, 2003 

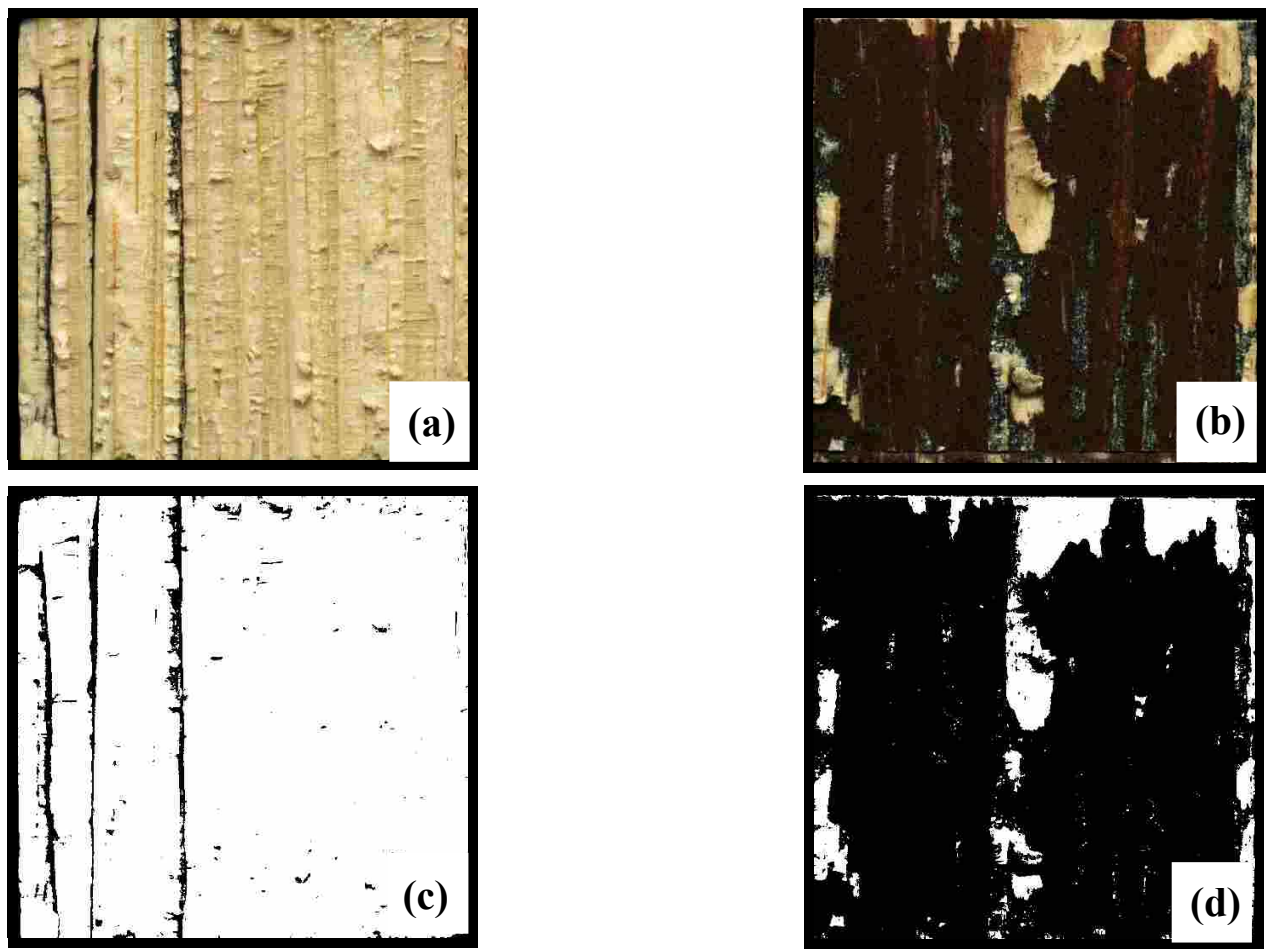

Figura 3 - Imagens originais (a e b) e binárias (c e d) de corpos-de-prova com alto (a e c) e baixo (b e d) porcentuais de falha na madeira.

Figure 3 - Original ( $a$ and $b$ ) and binary ( $c$ and $d$ ) test sample images with ( $a$ and $c$ ) high and (b and d) low wood failure.

Quadro 2 - Valores do estim ador $\hat{\beta}$, da diferença absoluta, do coeficiente de determ inação e do erro absoluto $m$ édio

Table 2 - Values of estim ator $\hat{\beta}$, absolute difference, coefficient of determ ination and $m$ ean absolute error

\begin{tabular}{|l|c|c|c|c|c|c|}
\hline & \multicolumn{7}{|c|}{ Tratamento } \\
\cline { 2 - 7 } & 1 & 2 & 3 & 4 & 5 & 6 \\
\hline$\hat{\beta}$ & 1,0198 & 1,1096 & 0,9883 & 0,8577 & 1,0159 & 0,9176 \\
DA & 0,0198 & 0,1096 & 0,0117 & 0,1423 & 0,0159 & 0,0824 \\
$\mathrm{r}^{2}$ & 0,9651 & 0,9668 & 0,9924 & 0,9574 & 0,9937 & 0,9966 \\
EMA (\%) & 7,52 & 10,45 & 3,94 & 10,20 & 3,19 & 4,39 \\
\hline
\end{tabular}

Quadro 3 - Análise da variância dos erros m édios absolutos dos algoritm os testados

Table 3 -Variance analysis of the m ean absolute errors of the algorithm $s$ tested

\begin{tabular}{|l|r|c|c|c|}
\hline \multicolumn{1}{|c|}{ FV } & GL & SQ & QM & F \\
\hline Tratamentos & 5 & 1046,070 & 209,2141 & $7,55^{* *}$ \\
Resíduo & 114 & 3159,599 & 27,71578 & \\
Total & 119 & 4205,670 & & \\
\hline
\end{tabular}

** significativo a $1 \%$ de probabilidade.

R. Árvore, Viçosa-MG, v.27, n.1, p.71-78, 2003
Os resultados do teste de Tukey para os valores dos erros médios absolutos estão no Quadro 4. Verificase que o tratamento 5 foi o que apresentou menor erro médio absoluto. Contudo, não houve diferença significativa pelo teste de Tukey, a 1\% de probabilidade, entre os erros médios absolutos dos tratamentos 1, 6, 3 e 5.

A Figura 4 ilustra a dispersão em torno de uma

Q uadro 4 - Resultado do teste de Tukey para o EM A Table 4 - $R$ esu lt of the $T$ ukey test for $E M A$

\begin{tabular}{|c|c|}
\hline Tratamento & EMA $^{*}$ \\
\hline 2 & $10,4544^{\mathrm{A}}$ \\
4 & $10,2029^{\mathrm{A}}$ \\
1 & $7,5212^{\mathrm{AB}}$ \\
6 & $4,3901^{\mathrm{B}}$ \\
3 & $3,9364^{\mathrm{B}}$ \\
5 & $3,1882^{\mathrm{B}}$ \\
\hline
\end{tabular}

* Médias seguidas pela mesma letra não diferem entre si pelo teste de Tukey, a $1 \%$ de probabilidade. 

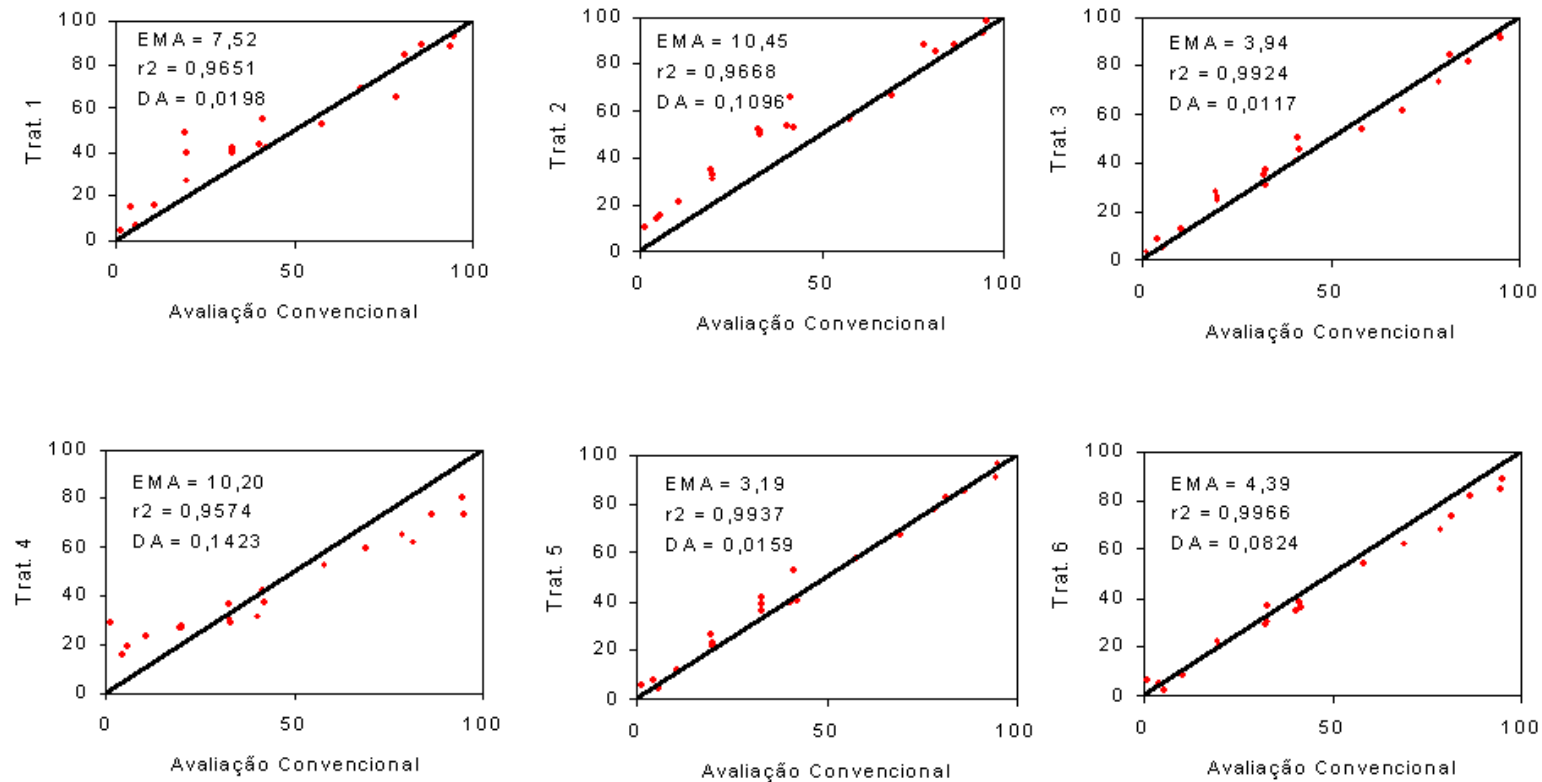

Figura 4 - Dispersão em torno da reta $\mathrm{y}=\mathrm{x}$ dos valores obtidos pela avaliação convencional versus os obtidos com os algoritmos propostos.

Figure 4 - Scattered plot of actual value against estimated value.

reta $\mathrm{y}=\mathrm{x}$ dos valores obtidos pela avaliação convencional versus os obtidos com os algoritmos propostos. Observa-se que os tratamentos 3 e 5 foram os que apresentaram melhor ajuste em torno dessa reta. O tratamento 6, apesar de ter apresentado EMA pequeno e um alto $\mathrm{r}^{2}$, teve seu ajuste prejudicado pela DA $(0,0824)$ em relação aos tratamentos $3(0,0117)$ e $5(0,0159)$.

\section{CONCLUSÕES}

a) A falha na madeira pode ser quantificada por limiarização automática em substituição ao método convencional das quadrículas.

b) O tipo de imagem e o modelo de digitalizador influenciaram os resultados obtidos pelos algoritmos para quantificação da falha na madeira.

c) Na quantificação da falha na madeira o algoritmo bayeseano apresentou resultados mais próximos aos da referência - método da quantificação da falha por contagem das quadrículas -, quando empregadas imagens coloridas adquiridas com o digitalizador Genius Color Page Vivid III.

d) $\mathrm{O}$ algoritmo iterativo apresentou resultados mais próximos aos das referências, quando empregadas imagens em tons de cinza adquiridas com o digitalizador
Genius Color Page Vivid III.

\section{REFERÊNCIAS BIBLIOGRÁFICAS}

AMERICAN SOCIETY FOR TESTING MATERIALS ASTM. Annual book of ASTM standards. Section 20 . Volume 15.06 - Adhesives. West Coshohocken, 2000. $600 \mathrm{p}$.

DELLA LUCIA, R. M.; VITAL, B. R. Avaliação da qualidade de juntas coladas de madeira de 3 espécies de Eucalyptus. Revista Árvore, v. 5, n. 2, p. 172-180, 1981.

GONZALEZ, R. C; WOODS, R. E. Processamento de imagens digitais. São Paulo: Edgard Blucher, 2000. 509 p.

HAN, Y. J.; HAYES, J. C. Soil cover determination using color image analysis. Transactions of the ASAE, v. 33, n. 4, p. 1402-1408, 1990.

HANEY, L. et al. Color matching of wood with a realtime machine vision system. ASAE, 1994. (ASAE Meeting Paper, 943579)

LIRA JR., M. A.; SMITH, D. L. Use of a standard TWAIN scanner and software for nodule number determination on different legume species. Soil Biology \& Biochemistry, v. 32, p. 1463-1467, 2000.

R. Árvore, Viçosa-MG, v.27, n.1, p.71-78, 2003 
MATEUS, G. R.; LUNA, H. P. Programação não linear. Belo Horizonte: Gráfica Formato, 1986. 289 p.

QUIN Jr., F.; STEELE, P. H.; SHMULSKY, R. Locating knots in wood with an infrared detector system. Forest Products Journal, v. 48, n. 10, p. 80-84, 1998.
REID, J. F.; SEARCY, S. W.; BABOWICZ, R. J. Determining a guidance in row crop image. ASAE, 1985. (ASAE Meeting Paper Paper, 85.3549).

VIEIRA-JUNIOR, P. A. et al. Emprego da técnica de análise de imagens na determinação do comprimento e da largura de sementes de milho. Revista Brasileira de Sementes, v. 21, n. 2, p. 110-119, 1999.

YANG, X. et al. Evaluation of Biofilm Image Thresholding Methods. Watter Research, v. 35, p. 1149-1158, 2001. 